\title{
Apocynin activity in spontaneously hypertensive rats (SHR): preliminary studies in vivo
}

\author{
R. Ciarcia -S. Damiano • C. Panico • R. Scanni • \\ F. Fiorito $•$ S. Florio $•$ J. W. Welch
}

Published online: 18 May 2010

(C) Springer Science+Business Media B.V. 2010

\begin{abstract}
An elevation in angiotensin II (Ang II) levels is a common occurrence in spontaneously hypertensive rats (SHRs). Infusions of Ang II and a high salt diet increase the activity of NADPH oxidase that stimulates superoxide anion $\left(\mathrm{O}^{-2}\right)$ generation and increases the expression of certain subunits of NADPH oxidase. Apocynin, an NADPH oxidase inhibitor with antihypertensive effects, is able to inhibit the release of superoxide anion by inhibiting NADPH oxidase activity and blocking the migration of p47 phox to the mitochondrial membrane. The aim of our study was to evaluate the antihypertensive effects of apocynin in SHRs and Wistar rats (WKYs) using a micropuncture technique. After microperfusion of both the proximal and distal tubules, we found that SHRs treated with apocynin showed a decrease in the free-flow collection of the proximal tubule (PT), which was not affected in WKYs. Moreover, significant differences were not demonstrated in the distal tubule (DT), probably due a mechanism of compensation that occurs in the loop of Henle. In conclusion, it is possible that the mechanisms of reabsorption in the PT are controlled by the interactions of $\mathrm{O}^{-2}$ and nitric oxide (NO). These data could suggest a higher activity of NADPH oxidase and increase in reactive oxygen species (ROS) production in the PT during hypertension.
\end{abstract}

Keywords Apocynin · Hypertension · Microperfusion · Kidney

R. Ciarcia $(\bowtie) \cdot$ S. Florio

Dipartimento Strutture Funzioni e Tecnologie Biologiche, Facoltà di Medicina Veterinaria,

Università degli Studi di Napoli "Federico II", Napoli, Italy

e-mail: roberto.ciarcia@unina.it

S. Damiano - R. Scanni

Dipartimento di Medicina interna, Sezione nefrologia, Facoltà di Medicina,

Seconda Universita' di Napoli, Napoli, Italy

C. Panico $\cdot$ J. W. Welch

Department of Medicine, Georgetown University, Georgetown, USA

F. Fiorito

Dipartimento di Patologia e Sanità Animale, Facoltà di Medicina Veterinaria,

Università degli Studi di Napoli “ Federico II”, Napoli, Italy 


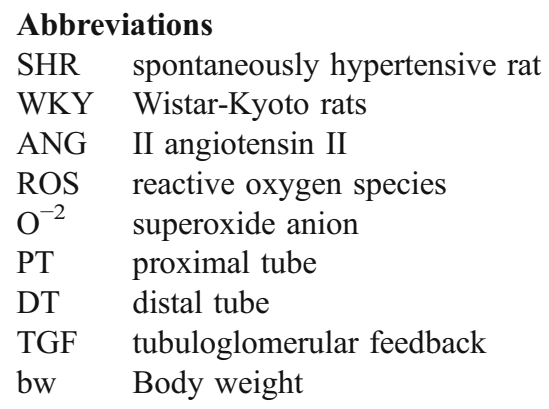

\section{Introduction}

SHRs are spontaneously hypertensive rats in which an elevation of angiotensin II (ANG II) level is a common occurrence. Harrison et al. (2003) have shown that the hypertension induced by ANG II correlates with oxidative stress, implying an increase of reactive oxygen species (ROS). Infusions of ANG II (Wang et al. 2003) and high salt diets (Kitiyakara et al. $2003)$ increase the activity of NADPH oxidase that stimulates superoxide anion $\left(\mathrm{O}^{-2}\right)$ generation and also improved the expression of some subunits of NADPH oxidase (p22 phox and p47 phox proteins). Furthermore, the involvement of NADPH oxidase during hypertension was observed, where the $\mathrm{p} 47$ phox protein appears to be an important factor for the generation of ROS (Babior 1999). NADPH oxidase is widely expressed in the kidney, and is present in blood vessels, interstitial cells, glomerulus, and tubules. The pathophysiological importance of this enzyme has led many researchers to use some NADPH oxidase inhibitors, in particular apocynin, also known as acetovanillone [4hydroxy-3-methoxyacetophenone], a natural organic compound with multiple pharmacological properties. Apocynin has antihypertensive effects. It is able to inhibit release of superoxide anions by inhibition of NADPH oxidase activity and blocks migration of p47 phox to the mitochondrial membrane (Babior 2004). $\mathrm{O}^{-2}$ generated by NADPH oxidase could be used for the production of ROS used by phagocytes or $\mathrm{O}^{-2}$ could be used as a second messenger in non-phagocytic cells ( $\mathrm{Li}$ et al. 2005). Due to the selectivity of its inhibition, apocynin could be widely used as an inhibitor of NADPH oxidase without interfering with other aspects of the immune system. The aim of this study was to evaluate in vivo the antihypertensive effects of apocynin in SHR and Wistar-Kyoto rats (WKY) rats using a micro-perfusion technique.

\section{Materials and methods}

Sixteen male SHR and 16 male WKY rats weighting $150 \pm 15 \mathrm{~g}$ were housed under constant environmental conditions of $22^{\circ} \mathrm{C}$ with a 12-h light-dark cycle. Rats were subdivided into four groups, each consisting of 8 animals, and treated for $24 \mathrm{~h}$ as follows: Group I SHR control; Group II - WKY control; Group III - SHR treated with apocynin orally (16 mg/Kg/die); Group IV - WKY treated with apocynin orally $(16 \mathrm{mg} / \mathrm{Kg} / \mathrm{die})$. The rats were anesthetized intraperitoneally with thiobarbital (Inactin; Sigma Aldrich, St. Louis, MO) $(120 \mathrm{mg} / \mathrm{kg} / \mathrm{bw})$ and prepared for the micro-puncture study of the left kidney using a thermo-regulated table $\left(37^{\circ} \mathrm{C}\right)$. For the tracheotomies, the jugular veins were catheterized with polyethylene PE-50 tubing that was used for intravenous infusion of saline solution 
Fig. 1 Blood pressure values in SHR and in WKY before and later the treatment. Data represents mean \pm S.D. of 8 rats. $* \mathrm{P}<0,0015$ vs. control. SHR have a significant increase of blood pressure correlated with WKY

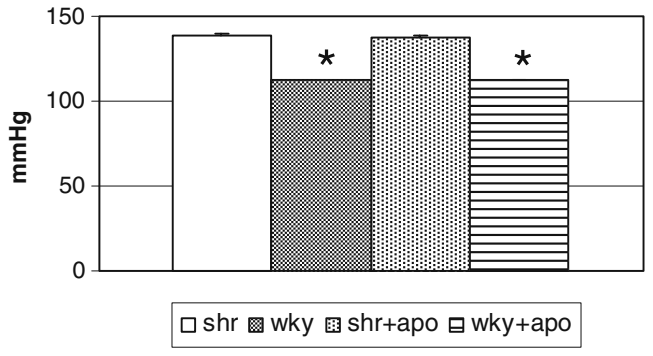

with an infusion pump (Braun, Melsungen, Germany). The femoral artery was catheterized to record the mean blood pressure with a pressure transducer (Powerlab, AD Instruments Inc, Colorado Springs, CO, USA). The bladder and ureter were catheterized with PE 90 and PE 10 polyethylene tubes, respectively, for collecting urine to evaluate kidney functionality. The left kidney was exposed by a flank incision and stabilized in a Lucite cup. After 45 min of equilibration, a micropipette containing mineral oil was inserted between the first and third loop of the proximal tubule. A drop of this oil was injected into the proximal tubule to follow the course of the flow and to make the proximal tubule block. The free flow collections of the medium proximal tubule and distal tubule were performed for a period of 3-4 min. The samples were then transferred to the capillaries and measured by calibrated microscope.

\section{Results}

Blood pressure and urinary flow

Systolic blood pressure was $139 \pm 0.57 \mathrm{~mm} \mathrm{Hg}$ in SHR and $112 \pm 11.2 \mathrm{~mm} \mathrm{Hg}$ in WKY rats $(\mathrm{p}<0.0015)$. Apocynin treatment did not induce any significant changes. Urine flows were similar in all groups.

Free-flow collections of proximal tubule (PT) and distal tubule (DT)

PT flow was similar in SHR as compared to normotensive rats $(14.01 \pm 2.7 \mathrm{nl} / \mathrm{min}$ in SHR, $12.01 \pm 3.6 \mathrm{nl} / \mathrm{min}$ in WKY). However, PT flow was substantially reduced in SHR treated with apocynin $(6.97 \pm 1.7 \mathrm{nl} / \mathrm{min})$ as compared to WKY treated with apocynin $(14.59 \pm$ $3.2 \mathrm{nl} / \mathrm{min}$ ). Additionally, there were not significant differences of DT flow in SHR and
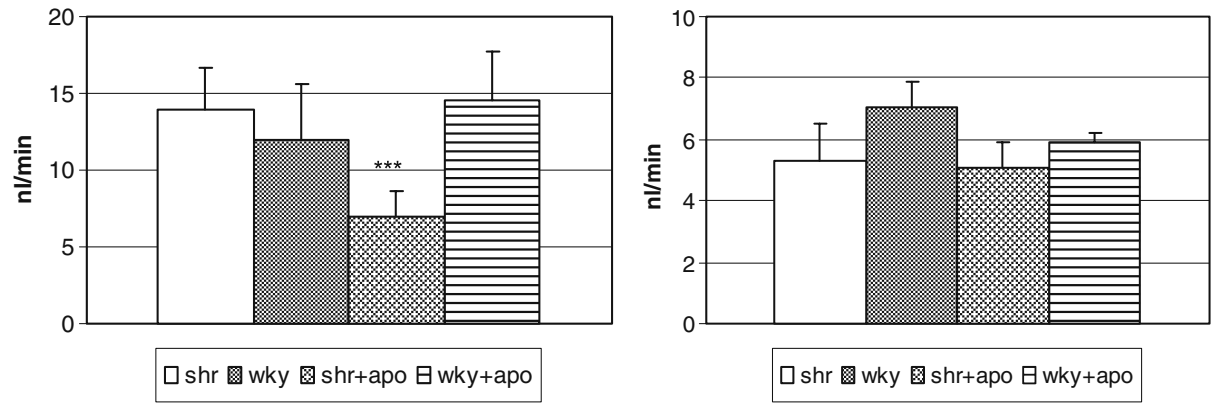

Fig. 2 Free flow collections of the proximal (left panel) and distal tube (right panel). Data represent mean \pm S.D 
WKY before or after treatment (SHR5.32 $\pm 1.2 \mathrm{nl} / \mathrm{min}$, WKY $7.05 \pm 0.8 \mathrm{nl} / \mathrm{min}$, SHR with Apo $5.08 \pm 0.8 \mathrm{nl} / \mathrm{min}$, and WKY with Apocynin $5.92 \pm 0.3 \mathrm{nl} / \mathrm{min}$ ).

This preliminary study shows that SHR rats treated with apocynin had decreased free flow in the proximal tubule, while significant difference was possible to see in WKY rats. In the distal tubule there were no differences in flow between all groups. This reduction in the proximal tubule was potentially compensated due to tubuloglomerular feedback (TGF) activation (Welch and Wilcox 1992).

\section{Discussion}

One new finding in this preliminary study was that PT flow is similar in SHR and WKY rats, which correlates with previous studies (Arendshorst and Beierwaltes 1979). The failure to detect basal differences in PT flow during hypertension remains unclear. This could be due to the method used in this study which blocks flow to the macula densa and tends to increase the glomerular filtration rate (GFR) and therefore, PT flow in both strains. Direct measurements of absolute fluid reabsorption by in situ microperfusion is the most accurate method to characterize PT function, since this method separates transport from the confounding effects of PT flow and TGF. Apocynin directly blocked NADPH oxidase, the most important source of $\mathrm{O}^{-2}$. Treatment with this drug significantly reduced the PT flow in SHR.

The aim of this work was to investigate the involvement of the NADPH oxidase during hypertension. Our data show that acute treatment with apocynin significantly reduced the proximal tubule flow. It is possible that the mechanisms of reabsorption in PTs are controlled by the interactions of $\mathrm{O}^{-2}$ and nitric oxide (NO). Renal levels of $\mathrm{O}^{-2}$ and NADPH oxidase are higher in SHR as compared with WKY rats (Welch et al. 2001). In conclusion, these data suggest an increased activity of NADPH oxidase and increased ROS production in the proximal tubule during hypertension (Figs. 1 and 2).

Acknowledgement Research was funded by MIUR (2007).

\section{References}

Arendshorst WJ, Beierwaltes WH (1979) Renal tubular reabsorption in spontaneously hypertensive rats. Am J Physiol 237:38-47

Babior BM (1999) NADPH oxidase: an update. Blood 93:1464-1476

Babior BM (2004) NADPH oxidase. Curr Opin Immunol 16:42-47

Harrison DG, Cai H, Landmesser U, Griendling KK (2003) Interactions of angiotensin II with NAD(P)H oxidase, oxidant stress and cardiovascular disease. J Renin-Angiotensin-Aldosterone System 4:51-61

Kitiyakara C, Chabrashvili T, Chen Y, Blau J, Karber A, Aslam S, Welch WJ, Wilcox CS (2003) Salt intake, oxidative stress, and renal expression of NADPH oxidase and superoxide dismutase. J Am Soc Nephrol $14: 2775-2782$

Li N, Zhang G, Yi FX, Zou AP, Li PL (2005) Activation of NAD(P)H oxidase by outward movements of $\mathrm{H}+$ ions in renal medullary thick ascending limb of Henle. Am J Physiol Renal Physiol 289:10481056

Wang D, Chen Y, Chabrashvili T, Aslam S, Borrego Conde LJ, Umans JG, Wilcox CS (2003) Role of oxidative stress in endothelial dysfunction and enhanced responses to angiotensin II of afferent arterioles from rabbits infused with angiotensin II. J Am Soc Nephrol 14:2783-2789

Welch WJ, Wilcox CS (1992) Potentiation of tubuloglomerular feedback in the rat by thromboxane mimetic. Role of macula densa. J Clin Invest 89:1857-1865

Welch WJ, Baumgärtl H, Lübbers D, Wilcox CS (2001) Nephron pO2 and renal oxygen usage in the hypertensive rat kidney. Kidney Intl 59:230-237 